\title{
Performance of Differenced Range Data Types in Voyager Navigation
}

\author{
T. H. Taylor, J. K. Campbell, R. A. Jacobson, \\ B. Moultrie, R. A. Nichols Jr., and J. E. Riedel \\ Navigation Systems Section
}

\begin{abstract}
Voyager radio navigation made use of a differenced range data type for both Saturn encounters because of the low declination singularity of doppler data. Nearly simultaneous two-way range from two-station baselines was explicitly differenced to produce this data type. Concurrently, a differential VLBI data type (DDOR), utilizing doubly differenced quasar-spacecraft delays, with potentially higher precision was demonstrated. Performance of these data types is investigated on the Jupiter-to-Saturn leg of Voyager 2. The statistics of performance are presented in terms of actual data noise comparisons and sample orbit estimates. Use of DDOR as a primary data type for navigation to Uranus is discussed.
\end{abstract}

\section{Introduction}

Spacecraft navigation during the Saturn encounters of Voyagers 1 and 2 was made more demanding by solar system geometry. For both spacecraft $(\mathrm{S} / \mathrm{C})$, late cruise and encounter occurred when the planet was near the line of nodes and thus at a geocentric declination of nearly zero (see Fig. 1). This caused special problems in $\mathrm{S} / \mathrm{C}$ orbit determination using radio data because of the so called "low declination singularity": use of conventional doppler and range data in this geometry leaves the $\mathrm{S} / \mathrm{C}$ declination poorly determined. Determination of right ascension, on the other hand, remains quite good when using conventional data.

No previous deep space mission had to contend with long periods of low declination, especially at such a critical time. To answer the potential loss of information, Voyager became the first mission to utilize explicitly differenced range as a basic data type. A fuller titular description might be "nearly simultaneous two-station two-way differenced range," but for simplification it will be referred to here simply as differenced two-way range (DTR). As will be discussed, DTR relieves the declination problem by differencing data between two stations on an intercontinental baseline having an appreciable northsouth projection. Because of this two-station feature, as well as other similarities with Very Large Baseline Interferometry (VLBI), DTR has also been called a "quasi VLBI" data type. DTR was adopted as the operational answer to the low declination problem.

Potentially more accurate than DTR is the true VLBI-based data type called "delta differential one-way range"(DDOR). As the name implies, this data type involves the differencing of one-way range from the spacecraft to each of two stations on an intercontinental baseline. The process is then repeated for a nearby quasar. Finally, these results are further differenced to provide a high accuracy spacecraft position with respect to the quasar. As with DTR, a declination determination depends on having a baseline with a large projection in the north-south 
direction. DDOR was an experimental data type on the Jupiter-to-Saturn cruise legs of both Voyager 1 and Voyager 2, and was to be further demonstrated in the cruise of Voyager 2 beyond Saturn to Uranus. However, performance to date has been such that it is now to become an operational data type (in August 1982) for the remainder of the Uranus cruise.

We will give a brief description of the salient features of each of the radio data types used in Voyager navigation during the approximately 2-year Voyager 2 cruise from Jupiter to Saturn. Features of the data arc and trajectory are discussed, followed by analyses of solutions on this arc.

\section{Description of Data Types}

Four distinct data types are discussed: doppler, range, DTR, and DDOR.

\section{A. Conventional Data (Doppler and Range)}

"Two-way" doppler data are normally the workhorse of JPL deep space navigation. The uplink carrier frequency from a Deep Space Station is detected and coherently retransmitted by the spacecraft. Upon receipt of this downlink signal, frequency is detected and differenced with what was transmitted a "round-trip light time" earlier. This constitutes the doppler information. Typically, one-minute averaged samples are sufficient to resolve Voyager line-of-sight velocity to within a few tenths of $1 \mathrm{~mm} / \mathrm{sec}$.

Range data are acquired by phase modulating the uplink signal with a series of pure square wave tones. The spacecraft turns this modulation around, and the receiving station then correlates these tones with a local model to obtain a phase shift. This phase shift is equivalent to measurement of the total propagation delay, uplink and downlink, at the station. In the absence of strong media noise (e.g., more than 30 or 40 degrees away from a solar conjunction), 5 minutes of correlation is sufficient to resolve Voyager range to the 1 -meter level. Absolute accuracy is typically at the 30-meter level.

Doppler and range data together yield both components of spacecraft state in the line-of-sight direction. In addition, angular information is obtainable from parallax effects resulting from motions of the earth, the spacecraft, and the earth's rotation.

Hamilton and Melbourne (Ref. 1) analyzed the information content for a single pass of doppler data from a single tracking station. Angular information is contained in the sinusoidal modulation of the earth's rotation onto range rate (doppler) (see Fig. 2). Right ascension (R.A.) is well determined from the phase of this modulation, while declination is determined by its amplitude. The accuracy of R.A. is relatively unaffected by spacecraft or tracking station positions. On the other hand, declination accuracy is very poor when $\mathrm{S} / \mathrm{C}$ declination is near zero because amplitude varies as the cosine of declination.

The amplitude is approximately given by:

$$
A=\omega r_{s} \cos \delta
$$

where

$$
\begin{aligned}
& \omega=\text { earth's rotation rate } \\
& r_{s}=\begin{array}{l}
\text { spin radius (distance from polar axis) of tracking } \\
\text { station }
\end{array} \\
& \delta=\text { spacecraft declination }
\end{aligned}
$$

This leads to an expression for accuracy of the estimated declination:

$$
\sigma_{\delta}=\frac{\sigma_{r_{s}}}{r_{s} \tan \delta}
$$

This is the source of the term "low declination singularity." It is apparent that spin radius errors (or anything which masquerades like them) can have a large effect on low declination solutions.

When multiple passes of data are used, angular content of the doppler is augmented by earth and spacecraft motion in heliocentric space. For long arcs, this effect can dominate over the earth rotation information, at least in certain directions. For Voyager, angular information is strongest in the ecliptic R.A. direction partially because the $S / C$ orbit is in the plane of the ecliptic. However, determination of geocentric declination is still fundamentally limited by the above considerations.

Conventional range data are generally inadequate to provide the same kind of angular information as doppler. Not only is range noisier, but inadequately modelled nongravitational accelerations (e.g., from minute gas leaks and from unbalanced thrusting of the attitude control system) can cause gross orbit errors when range is not used with care. For this reason it is usually used in combination with other data types, and is weighted much more loosely than its inherent accuracy would seem to warrant. Thus it usually provides only range information, while doppler provides range rate.

\section{B."Differenced Two-Way Range}

In the above discussion, attention was focused on single station tracking. When more than one station is used, doppler data are still hampered by the low declination problem. How- 
ever, when data are gathered from widely separated stations on a large earth baseline, conventional range data could in principle be used for accurate angular determination. Unfortunately, the inadequately modelled acceleration problem still pertains and, additionally, solar plasma becomes a major concern.

The differenced two-way range data type was created to deal with these problems. A DTR point is simply generated by explicitly differencing two conventional range points from widely separated stations. The differencing eliminates errors common to both stations such as unmodelled spacecraft accel. erations. If the conventional range points are close in time (simultaneous two-way ranging is not possible), then space plasma effects are also minimized.

A simplified geometric discussion loosely following Melbourne and Curkendall (Ref. 2) illustrates the ability of DTR to determine angles, and particularly declination. A delay measurement (e.g., a nearly simultaneous DTR point) provides S/C angular information by the relation

$$
\tau=S \cdot B=|B| \cos \theta
$$

where

$$
\begin{aligned}
& \tau=\text { the geometric differential delay } \\
& S=\text { the unit vector directed from the earth's center to the } \\
& \quad \text { radio source } \\
& B=\text { the baseline vector } \\
& \theta=\text { the angle between } B \text { and } S .
\end{aligned}
$$

In geocentric coordinates

$$
\tau=B_{e} \cos \delta \cos \left(\alpha_{B}-\alpha\right)+B_{z} \sin \delta
$$

where $B_{e}$ and $B_{z}$ are the baseline's equatorial and polar projections, respectively, $\alpha$ is the source's R.A., and $\alpha_{B}$ is the baseline's R.A. From this equation, the sensitivity of delay to declination as declination approaches zero is

$$
\frac{\partial \tau}{\partial \delta}=B_{z}
$$

That is, DTR retains sensitivity to declination in low declination geometries.

Figure 3 presents a simplified case of a baseline in the north-south direction. It is clear from this and from the above discussion that an expression of accuracy is given by

$$
{ }_{\delta}^{\sigma}=\frac{\sigma_{\Delta \rho}}{|B| \cos \delta}
$$

where $\sigma_{\Delta \rho}$ is the standard error in the difference of the two range measurements.

The NASA Deep Space Network (DSN) stations available for tracking are shown in Fig. 4. Notice that the Spain-Goldstone (S-G) baseline is nearly due east-west so that essentially no declination is available from that baseline. Declination is available primarily from the Goldstone-Australian (G-A) and Australia-Spain (A-S) baselines.

The range points chosen for explicit differencing are the last few at the end of the pass of the outgoing station and the first few of the incoming station. Ordinarily, the closest points are 15 minutes apart except for the A-S baseline where roundtrip light time near Saturn exceeded the mutual visibility period and the closest possible points were 2 hours and 15 minutes apart. This was large enough to allow solar plasma effects to become significant in some instances, and so the value of this baseline for DTR was questionable.

The Voyager requirement on DTR was 6.4 meters accuracy (1 sigma). This is equivalent to about 600 nanoradians accuracy on the G-A baseline.

\section{Delta Differential One-Way Range}

The above equations for DTR apply to any differenced range data type, and this, of course, includes VLBI. DDOR is a differential data type generated by explicitly differencing a one-way S/C VLBI delay measurement with the corresponding measurement for an angularly nearby quasar. A detailed description is given by Border et al. (Ref. 3). Thus DDOR has some of the same attributes as DTR, particularly the ability to determine declination in similar geometries. It also shares the feature of "self-calibration" by cancellation (through the implicit differencing of a VLBI measurement) of errors common to each baseline (e.g., common path space plasma errors). Additionally, however, the second (explicit) differencing of $\mathrm{S} / \mathrm{C}$ with quasar data allows further self-calibration in the cancellation of error sources such as dispersive transmission media, inaccurate station locations and earth platform parameters, and station clock differences. These features combined give DDOR potentially higher precision than DTR.

DDOR measurements typically require about a half-hour of tracking time: 10 minutes on the $S / C, 10$ on a quasar, and 10 minutes of antenna slewing time.

The expectation for DDOR accuracy on the Jupiter-toSaturn cruise leg of Voyager 2 was about $4 \mathrm{~m}$. This included allowance for $3.7 \mathrm{~m}$ of bias in quasar position. 


\section{Description of the Data Arc and Trajectory}

The data arc is almost 2 years long, beginning 13 November 1979, about 4 months after the Voyager 2 Jupiter encounter, and ending 4 August 1981, 22 days before the Saturn encounter. The goal was to incorporate as much of the differenced data as possible on a single trajectory rather than in disjointed sets. It was not possible to use the original operational set of trajectories since they consisted of a series of shorter arcs.

The epoch state for the trajectory was initially derived from an operational solution obtained early in 1981. Three large Trajectory Change Maneuvers (TCMs) were modeled, as well as 25 smaller impulsive maneuvers due to delta Vs incurred during $\mathrm{S} / \mathrm{C}$ attitude changes. Solar radiation pressure was modeled, but does not contribute significantly to the nongravitational acceleration profile at $5-10 \mathrm{AU}$ solar distance.

Processing the entire original data set was out of the question due to sheer volume (35,000 total range and doppler points) and the consequent computing costs. Also, there was a desire to see the effect of reducing conventional data to a minimum, letting the differenced data predominate. Thus, conventional data were severely edited, leaving about 670 doppler and 260 range points available. Data were selected so as to be evenly balanced among the three DSN sites. Doppler was selected at the rate of about 3 points per day (using 5 and 20 minute averaging periods), and range 1 point per day. These conventional data were calibrated for the effects of troposphere, ionosphere, and equipment group delays; but not for space plasma effects.

DTR was processed from a subset of the calibrated range data, from those tracking periods which had been scheduled specifically for generation of nearly simultaneous range points. Such periods were normally scheduled once each month, and usually consisted of 4 contiguous passes, beginning and ending at the Australian site. Thus 3 baselines were available each period. Generally, 4 points were chosen from each station for each baseline, and differencing order proceeded from the closest 2 points to the 2 most widely separated ones. Since points were 5 minutes apart, typical separations were 15 to 45 minutes for the S-G and G-A baselines, and from 2 hours 15 minutes to 2 hours 45 minutes for A-S. A total of 52 baselines was used, consisting of 203 differenced pairs.

DDOR was an experimental data type on this arc, and so coverage was more sporadic. Two baselines were used: S-G and G-A. Before February 1981, about 2 baselines per month were scheduled. After this, baselines were scheduled at an average rate of 1 every several days until encounter. During the entire trajectory, only 2 quasars were used: P1055+01 until 27 August 1980, and 3C273 thereafter. After editing out bad data, 58 DDOR points (baselines) remained. These were calibrated for troposphere and ionosphere.

The JPL Orbit Determination Program (ODP) was used for solutions, using a conventional weighted least squares batch sequential filter with no correlation between batches. Batch length was 30 days, yielding 22 batches in the data arc. Estimated quantities were $\mathrm{S} / \mathrm{C}$ state, bias and stochastic nongravitational accelerations, the earliest two TCMs, delta-Vs due to attitude changes, and quasar positions. Station location errors were considered for their effect on orbit uncertainty, but not estimated.

\section{Solutions and Results}

For evaluation of performance it is necessary to compare data types - directly, in terms of their residuals, and indirectly in terms of the orbit solutions they generate. Table 1 gives a list of the combinations of data types and weights used to generate five solutions of interest.

Range is used in all of these, weighted loosely at $100 \mathrm{~km}$. Doppler is used in three cases to provide strong determination of R.A. as well as highly accurate range rate. In the other two cases, doppler is left out and angles are determined solely by the differenced range data types.

Comparisons of solutions are made in the target planet "B-plane," illustrated in Fig. 5, where the B vector for the best current postencounter solution, the "reconstruction" solution, is plotted. Figure 6 compares the doppler solutions in the B-plane, relative to the above mentioned "best" solution. One-sigma error ellipses are drawn.

The most obvious feature is the disagreement in (ecliptic) declination between the doppler-range solution and solutions using differenced range. The differenced range solutions are in relatively good agreement in declination, each being within the other's error ellipse. The fact that all three agree in ecliptic R.A. is not surprising, since the doppler in each solution dominates the determination in that direction. Two solutions were done to entirely remove the influence of doppler by fitting only DDOR-range and DTR-range. They are shown in Fig. 7 along with error ellipses. Now the R.A. alignment is gone as each solution is allowed to float independently in that direction. The DDOR solution has moved north-east; and DTR, south-west. 
Figures 8 and 9 plot residuals from the two DDOR baselines from the DDOR-doppler-range solution. Table 2 presents means and calculated 1-sigma noise for each case.

The fit seems good on both baselines as compared to the expected 4-meter accuracy. There is the possibility, of course, that the filter is simply "fitting out" some of the noise. This speculation is belied, however, by the dense data in the period from day 456 to 484 . There, the noise on the S-G baseline is $0.6 \mathrm{~m}$, while the G.A noise is $1.0 \mathrm{~m}$. This span is too short for the filter to have significant effect, and so this result is commensurate with (in fact, better than) that of the entire data span.

DTR residuals are plotted in Figs. 10 through 12. Noise is good on two baselines. However, there is a poor fit on the A-S baseline. This one was expected to be poor due to the large time separation between points, allowing solar plasma variations to cause significant variations. The two points near -4 meters should probably have been deleted from the data set. However, they had little effect on the solution considering the total number of competing points on the S-G baseline. (The G-A baseline projection toward the $\mathrm{S} / \mathrm{C}$ is nearly orthogonal to the A-S projection.) This was confirmed in a subsequent solution in which they were removed: the B-plane changed less than $100 \mathrm{~km}$. (In fact, in another solution the entire A-S baseline was thrown out with, again, less than $100 \mathrm{~km}$ of difference in the B-plane.) Noise is quite good during the high-density period from day 576 through 611 $(1.1 \mathrm{~m}$ on $\mathrm{S} / \mathrm{G}$ and $1.2 \mathrm{~m}$ on $\mathrm{G} / \mathrm{A})$, making it unlikely that the filter is making noise overly optimistic over the entire arc.

The total separation between the two differenced range solutions (without doppler) is significant in terms of the residuals of those data types. To compare the solutions with the residuals, we use the DDOR residuals which were passed through in the DTR-range solution. They are plotted in Figs. 13 and 14 . There is a clear bias of about -5 meters (at the end of the arc) in the S-G direction and -8 meters in the G/A direction. These biases should point, in some sense, from the DTR solution to the DDOR solution. To test a simple vector representation in the B-plane, we plot these biases in the diagonal coordinates of the projection of the baselines, using the approximation of 1 meter equals 150 kilometers at Saturn. The result is shown in Fig. 15. The correspondence of B-plane relative positions with this vector treatment of the residuals is obviously not perfect, but it is good enough to allow some experimentation. It holds up as a rule of thumb on the other solutions already plotted, and so we can use it to get a rough idea of the absolute biases of each data type.

The DTR-range and DDOR-range solutions disagree with each other and-with the solutions containing doppler. Since doppler dominates in R.A., it is easy to believe that the correct solution probably lies near a line connecting the doppler solutions ${ }^{1}$. Taking the differenced range-dopplerrange solutions themselves as extremes for the "correct" solutions and resolving vectors into baseline coordinates, we get the results listed in Table 3. Thus, a regime of biases is roughly determined for each data type and baseline, predicated on the hypothesis that - in R.A. at least - doppler knows best. For example, Table 3 indicates that the probable bias for DDOR on the G-A baseline is between -3.5 and $-6.5 \mathrm{~m}$. For DTR on the G-A baseline, it is between 2.0 and $5.0 \mathrm{~m}$.

A single solution in the B-plane provides a limited picture of performance. Therefore, a history of solutions was formed for three of the data combinations already discussed by solving at 60-day (2-batch) intervals from the epoch, each interval containing all the information from epoch to the end of the corresponding batch. Figure 16 shows the B-plane history for doppler-range. This succinctly illustrates the weakness in declination. Figure 17 shows the DTR-range results. Note the change of scale. The tightening of the solution in declination can be seen in comparison to the doppler-range case. Results for the DDOR-range solutions are in Fig. 18. The tight declination is also seen here. Formal consider sigmas for these same cases are shown in Fig. 19 for B $\cdot \mathrm{T}$ and Fig. 20 for B $\cdot$ R. The overwhelming early dominance of doppler is seen in the B - T plot. DTR and DDOR do not come close to matching this performance until late in the data arc. One reason for this is, of course, the sparsity of the differenced range data types until early 1981; whereas doppler data were used more uniformly throughout the arc. Superiority of the differenced range data types in declination is clearly seen in Fig. 20. Early in the plot S/C declination is around $8 \mathrm{deg}$. In August 1980 (batch 10), declination goes below 5 deg, staying close to 0 from then on through the encounter. Declination at encounter is about $-2 \mathrm{deg}$. The effect on the doppler determination of $B \cdot R$ is apparent.

Comparison of the plots for the histories with plots for the uncertainties seems to show that the differential range types performed better than the curves would indicate. This may be due to larger than necessary a priori sigmas on nongravitational accelerations and impulsive maneuvers.

\footnotetext{
1The "correct" solution might be thought to be the reconstruction solution at the origin. This is fortuitously close to the DTR-dopplerrange solution. "Fortuitous," however, is the operative word, considering the size of the error ellipse and also the fact that the string of operational solutions which led to the reconstruction used primarily doppler, range, and DTR as the radiometric data types. The fact that the final reconstruction is close to the DTR solution simply expresses the fact that this data arc is compatible with the string of arcs which yielded the reconstruction. Given that compatibility, DTR is guaranteed to excel.
} 


\section{Discussion and Conclusions}

Noise performance of each differenced range data type was good. The estimated 1 -sigma noise for DDOR was $1.1 \mathrm{~m}$ overall, while DTR noise was $2.8 \mathrm{~m}$. However, as seen in Table 3, there were significant biases for each data type if the assumptions in the above section are correct. This is reinforced by data from the current operational Voyager 2 trajectory where the relative bias (from DTR to DDOR) is $-3.0 \mathrm{~m}$ on the S-G baseline and $-5.5 \mathrm{~m}$ on the G-A baseline. This is compared with values of $-5.0 \mathrm{~m}$ and $-8.0 \mathrm{~m}$ in the pre-Saturn trajectory. Thus, it appears that errors have remained about the same despite the use of new quasars and some changes in the ranging system since the encounter. Expected accuracies prior to encounter were about $4.0 \mathrm{~m}$ for DDOR and $6.4 \mathrm{~m}$ for DTR. Noise for both data types compares very favorably with these numbers, but the assumed biases are less favorable. It appears that DTR probably met expectations, but that DDOR may have performed more poorly than expected.

From another viewpoint, the low data noise is the most significant feature for navigational purposes. Low noise allows good determination of $\mathrm{S} / \mathrm{C}$ angular rate. It is this angular rate which will be the dominant feature in long mappings (e.g., from the present to the Uranus encounter), whereas positional errors will be relatively small and unchanged by mappings, especially considering the size of the Uranus ephemeris uncertainty which is the equivalent of about a $25-\mathrm{m}$ bias in the R.A. direction, and 5.5 in declination. From this standpoint, DDOR excels.

Since Saturn, DDORs have been accomplished in the higher frequency X-band rather than the pre-encounter S-band. This results in increased signal strength for the $\mathrm{S} / \mathrm{C}$ and decreased susceptibility to charged particle media errors. Additionally, the effective bandwidth has been increased. These have had the effect of decreased DDOR noise. The results are $0.6 \mathrm{~m}$ noise on the S-G baseline on 9 baselines since January 82, and an incredibly low $0.1 \mathrm{~m}$ on the $8 \mathrm{G}$-A baselines in the same period.

After having performed well on the Voyager 2 approach to Saturn, DTR was retired from Voyager service in March of 1982. Its use was becoming too unwieldy because of increased round-trip light times and decreasing station overlaps due to decreasing declination. Indeed, the round-trip requirement of DTR is perhaps its worst drawback. At the least, it causes operational difficulties, and at the worst it destroys the requirement of near simultaneity. At Uranus the round-trip light time of 5.5 hours will exceed the effective overlap on all DSN baselines. In contrast, DDOR (by definition) requires only a short mutual visibility period of quasar and spacecraft over about a half-hour.

Since the Saturn encounter, S/C declination has been decreasing and is presently passing through about $-10 \mathrm{deg}$. At Uranus, it will be near $-23 \mathrm{deg}$. In this region, doppler becomes competitive with DDOR in determination of declination and, at first glance, there would seem to be no reason to continue the use of DDOR. There are, however, a few good reasons to continue. One of these is the fact that the same round-trip light time which renders DTR impractical also significantly reduces the amount of conventional data which can be gathered during the available $\mathrm{S} / \mathrm{C}$ visibility periods at each station. At Spain, the tracking mode would have to be two-way for the entire 7-hour period (at Uranus) in order to receive 1.5 hours of data. At Goldstone, the margin is only 1 hour better. Only Australia will have a $\mathrm{S} / \mathrm{C}$ visibility period (12 hours) significantly greater than the round-trip light time.

However, extended periods of two-way operation are becoming objectionable as the $\mathrm{S} / \mathrm{C}$ distance increases because uplink noise begins to interfere with downlink telemetry. The downlink is phase-locked at a multiple of $11 / 3$ to the uplink frequency, and so $\mathrm{S} / \mathrm{C}$ received phase noise is actually amplified onto the downlink. Thus there is the desire to use less two-way data. DDOR is the ideal candidate to remedy this situation. This is especially true in light of the recently improved performance of DDOR. As a result, data accuracy for operational orbit determination is currently assumed to be $1 \mathrm{~m}$. This allows a few baselines of DDOR to replace a great deal of doppler in determining angular position and rates. The present schedule calls for 2 doppler-range single station passes per week (about 8 hours per pass; this is as compared to nearly continuous tracking on previous interplanetary legs) and 3 DDOR measurements per month. 


\section{References}

1. Hamilton, T. W., and Melbourne, W. G., "Information Content of a Single Pass of Doppler Data from a Distant Spacecraft," Space Program Summary 37-39, Vol. III, Jet Propulsion Laboratory, Pasadena, Calif., May 31, 1966.

2. Melbourne, W. G., and Curkendall, D. W., "Radio Metric Direction Finding: A New Approach to Deep Space Navigation," presented at AAS/AIAA Astrodynamics Specialist Conference, Jackson Hole, Wyoming, Sept. 7-9, 1977.

3. Border, J. S., Donivan, F. F., Hildebrand, C. E., Moultrie, B., Skjerve, L. J., and Finley, S. G., "Determining Spacecraft Angular Position with Delta VLBI: The Voyager Demonstration," presented at AIAA/AAS Astrodynamics Conference, San Diego, Calif., Aug. 9-11, 1982.

4. Curkendall, D. W., and Ondrasik, V. J., "Analytic Methods of Orbit Determination," presented at AAS/AIAA Astrodynamics Conference, Vail, Colo., July 16-18, 1973.

5. Siegel, H. L., Christensen, C. S., Green, D. W., and Winn, F. B., "On Achieving Sufficient Dual Station Range Accuracy for Deep Space Navigation at Zero Declination," presented at AAS/AIAA Astrodynamics Specialist Conference, Jackson Hole, Wyoming, Sept. 7-9, 1977. 
Table 1. Data combinations and weights

1. Doppler-range (Dop-Rng)

2. DDOR-doppler-range (DDOR-Dop-Rng)

3. DTR-doppler-range (DTR-Dop-Rng)

4. DDOR-range (DDOR-Rng)

5. DTR-range (DTR-Rng)

$\begin{array}{ll}\text { Doppler } & 1 \mathrm{~mm} / \mathrm{sec} \\ \text { Range } & 100 \mathrm{~km} \\ \text { DDOR } & 2 \mathrm{~m} \\ \text { DTR } & 5 \mathrm{~m}\end{array}$

Table 2. Noise and means

\begin{tabular}{lccccccc}
\hline & \multicolumn{3}{c}{ DDOR } & & \multicolumn{3}{c}{ DTR } \\
\cline { 2 - 4 } \cline { 6 - 8 } & Noise & Mean & Baselines & & Noise & Mean & Baselines \\
\hline S-G & 1.1 & 0.0 & 32 & & 2.5 & -0.5 & 18 \\
G-A & 1.1 & 0.0 & 26 & & 1.3 & 0.1 & 24 \\
A-S & - & - & - & & 4.3 & 3.4 & 10 \\
All & 1.1 & 0.0 & 58 & & 2.8 & 0.5 & 52 \\
\hline
\end{tabular}

Units $=$ meters

Table 3. Resolution of solutions into biases

\begin{tabular}{llllll}
\hline & \multicolumn{2}{c}{ DDOR } & & \multicolumn{2}{c}{ DTR } \\
\cline { 2 - 3 } \cline { 5 - 6 } & S-G & G-A & & S-G & G-A \\
\hline Ref. point & & & & \\
DDOR-DOP-RNG & -3.5 & -3.5 & & 3.0 & 5.0 \\
DTR-DOP-RNG & -4.0 & -6.5 & & 2.5 & 2.0 \\
\hline
\end{tabular}

Units $=$ meters 


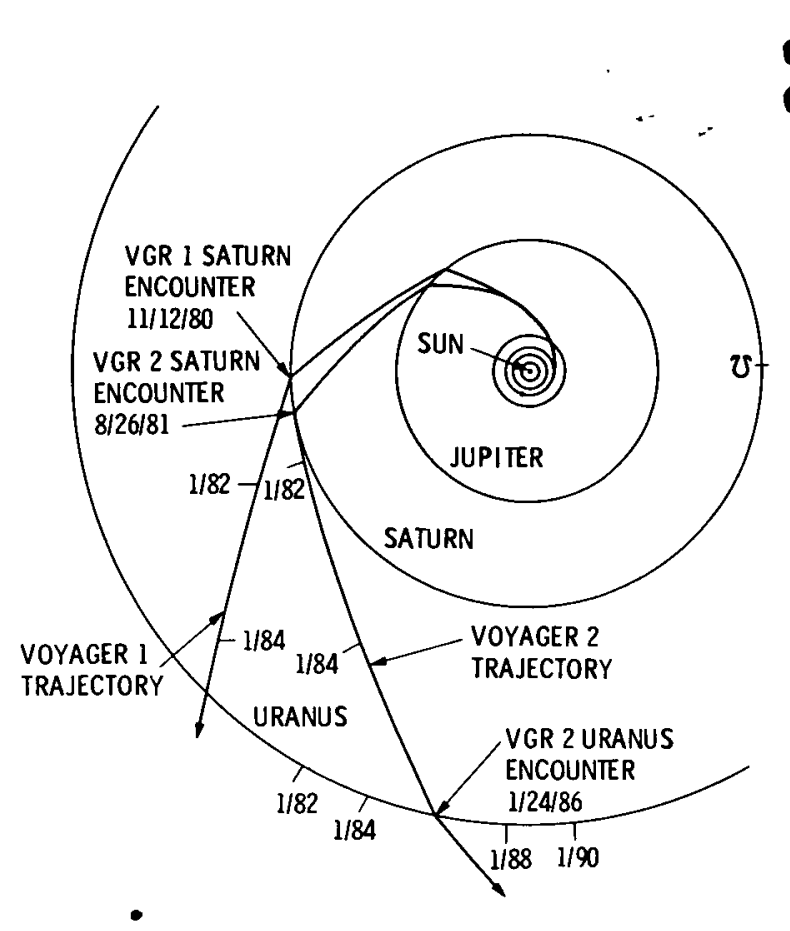

ORIGINAL PAGE IS
OF POOR: QUALITY

Fig. 1. Voyager trajectories
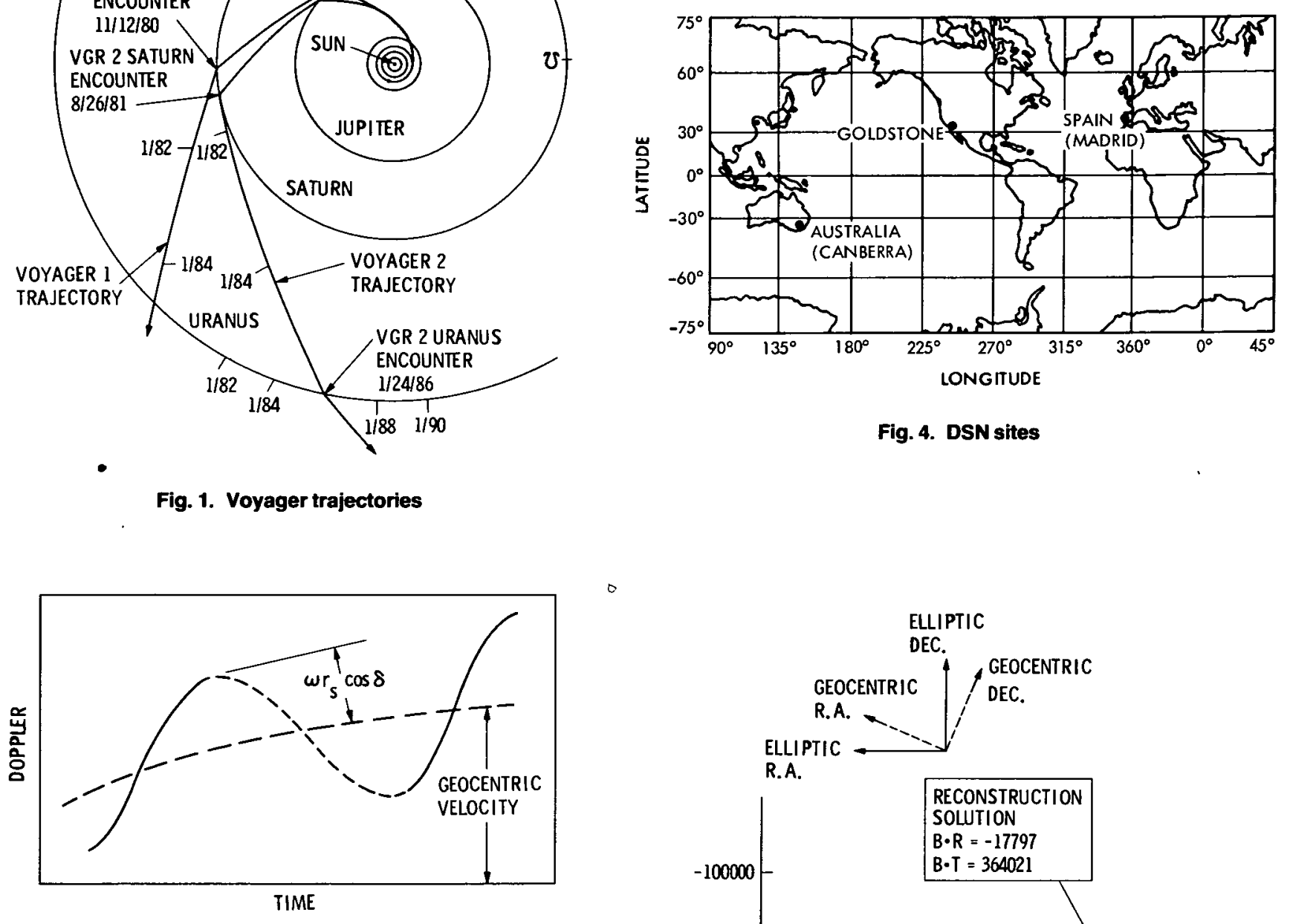

Fig. 4. DSN sites

Fig. 2. Doppler signature
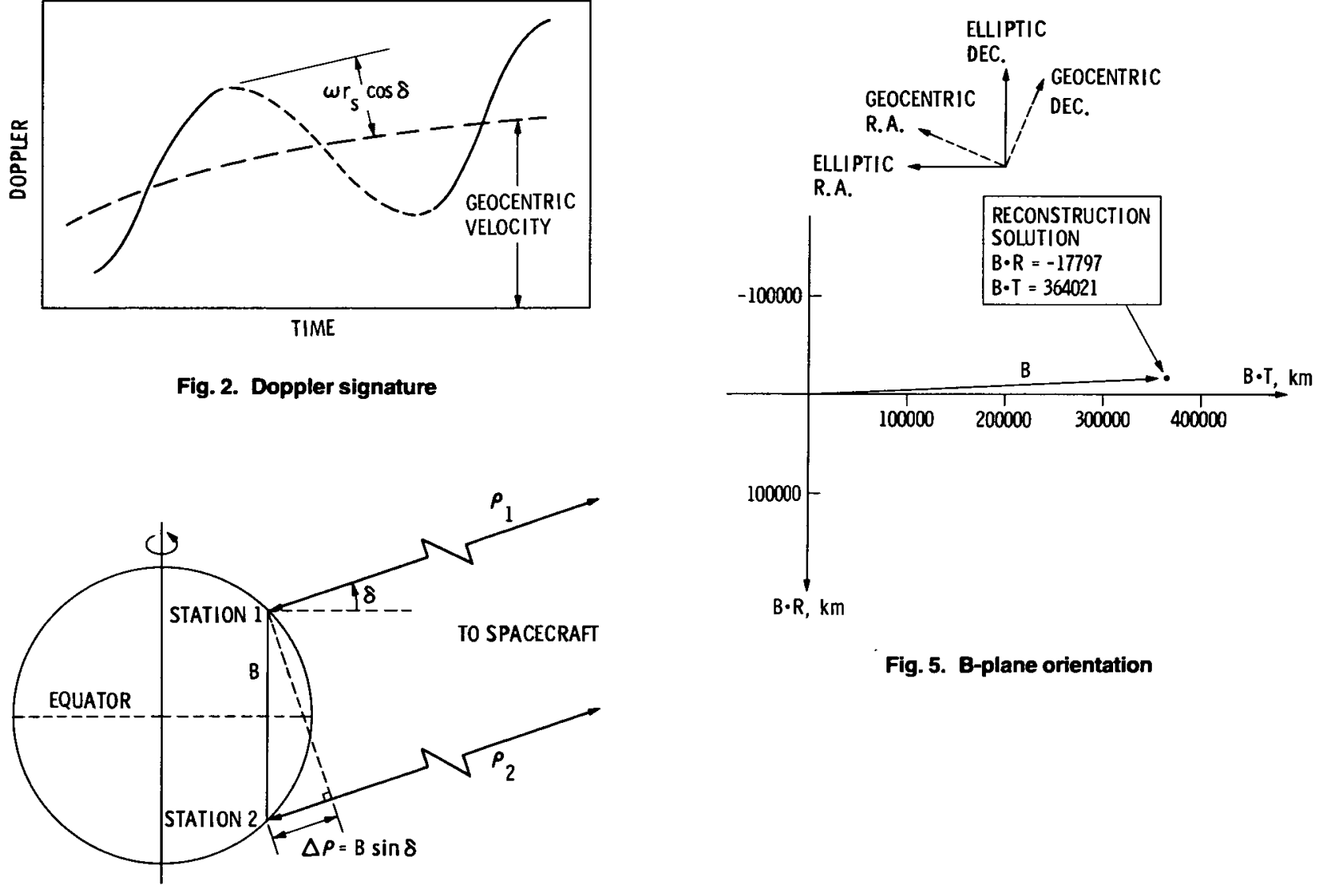

Fig. 5. B-plane orientation

Fig. 3. Ranging from two stations 


\section{ORIGNAL PAGE BG \\ OF POOR QUALTY}

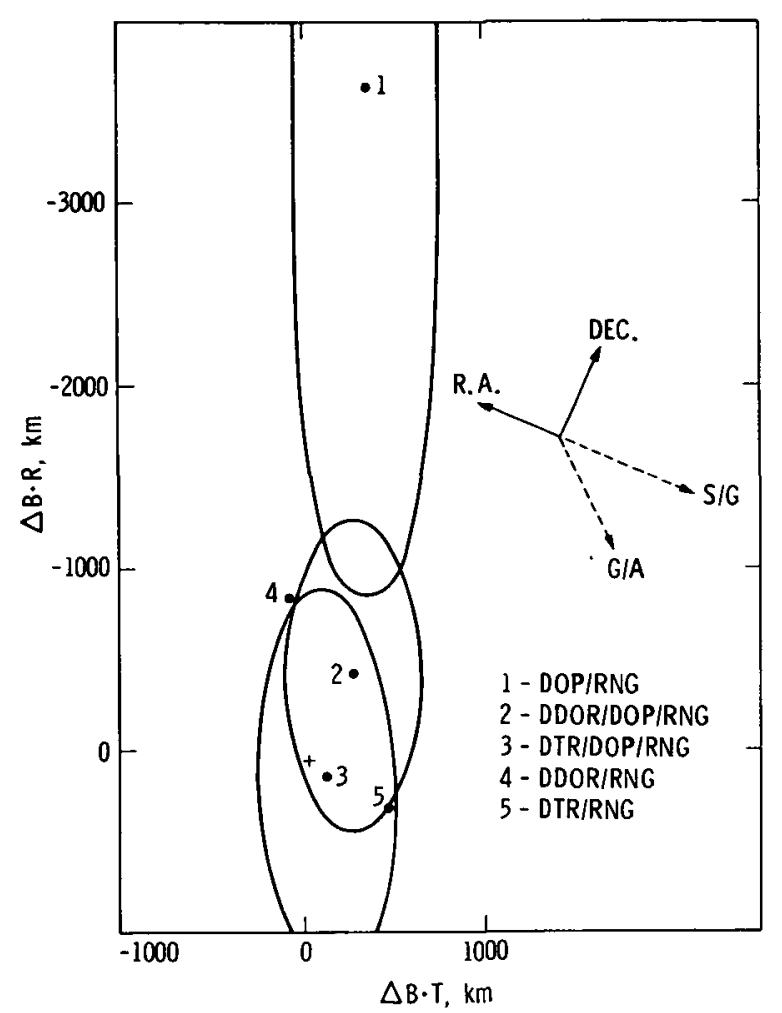

Fig. 6. B-plane solutions with doppler

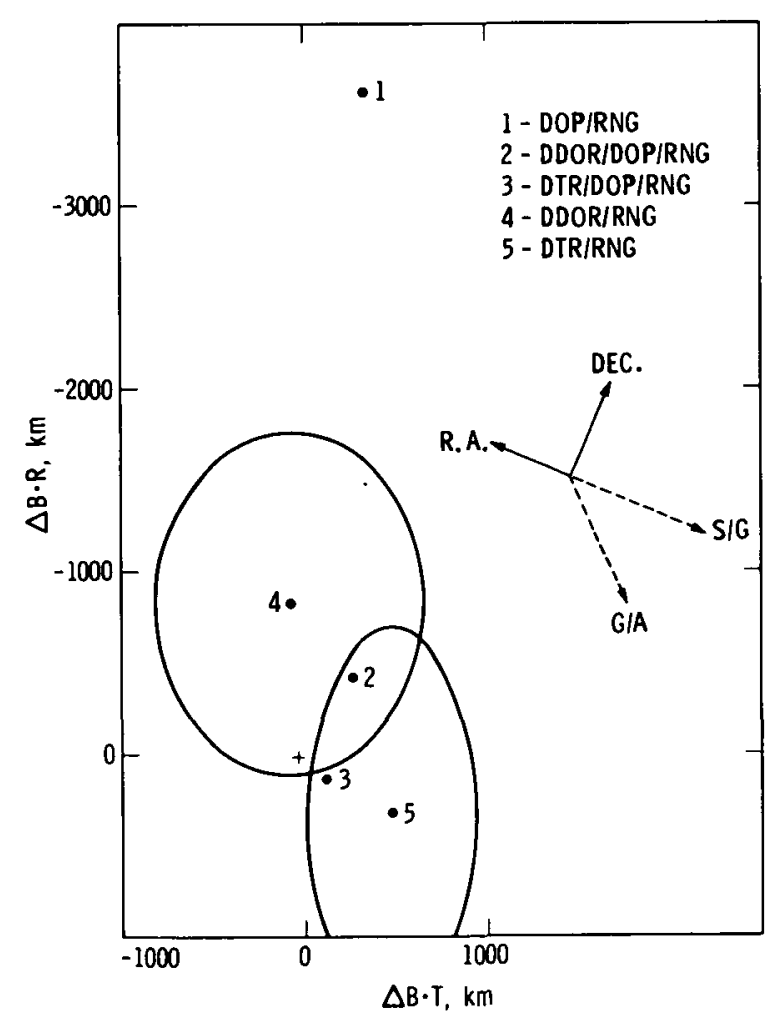

Fig. 7. B-plane solutions without doppler

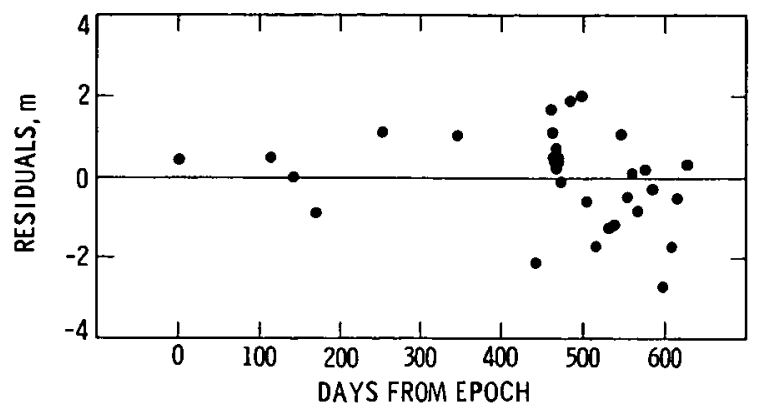

Fig. 8. DOOR residuals, S-G baseline

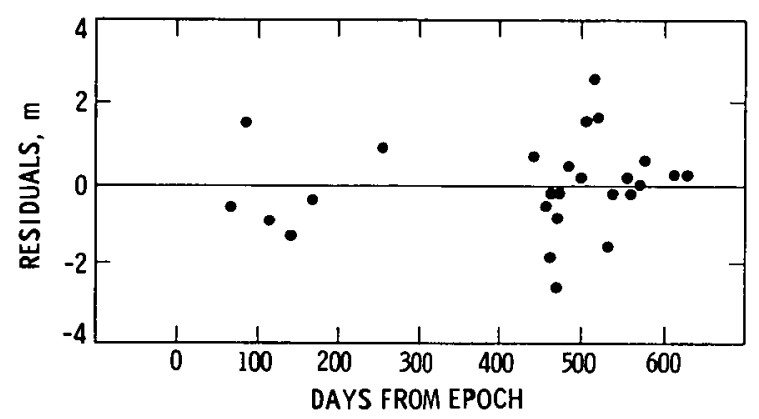

Fig. 9. DDOR residuals, G-A baseline

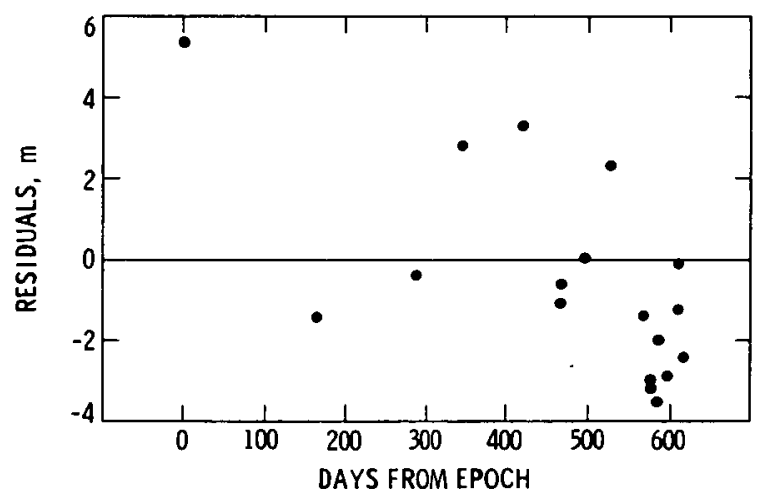

Fig. 10 DTR residuals, S-G baseline 


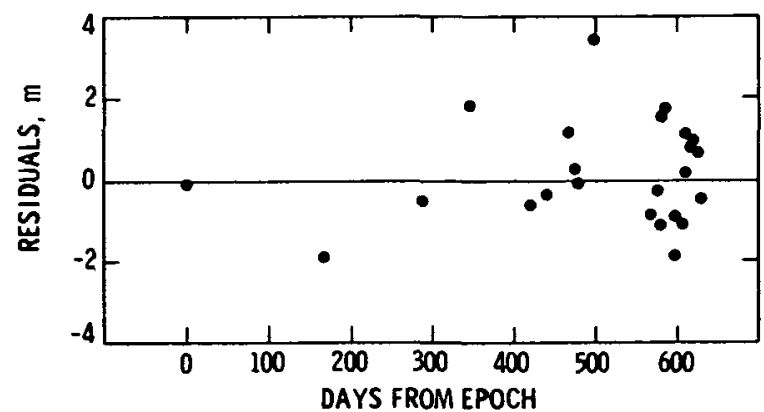

Fig. 11. DTR residuals, G-A baseline

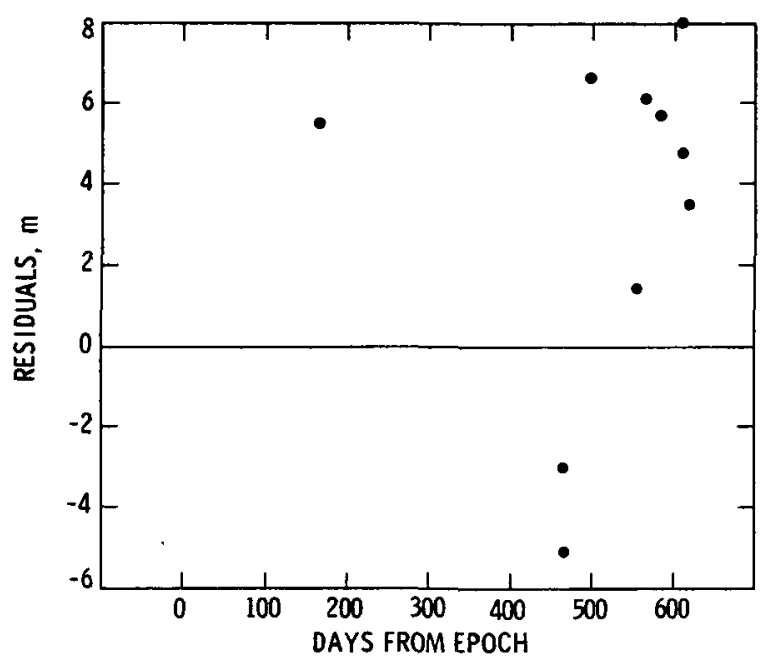

Fig. 12. DTR residuals, A-S baseline

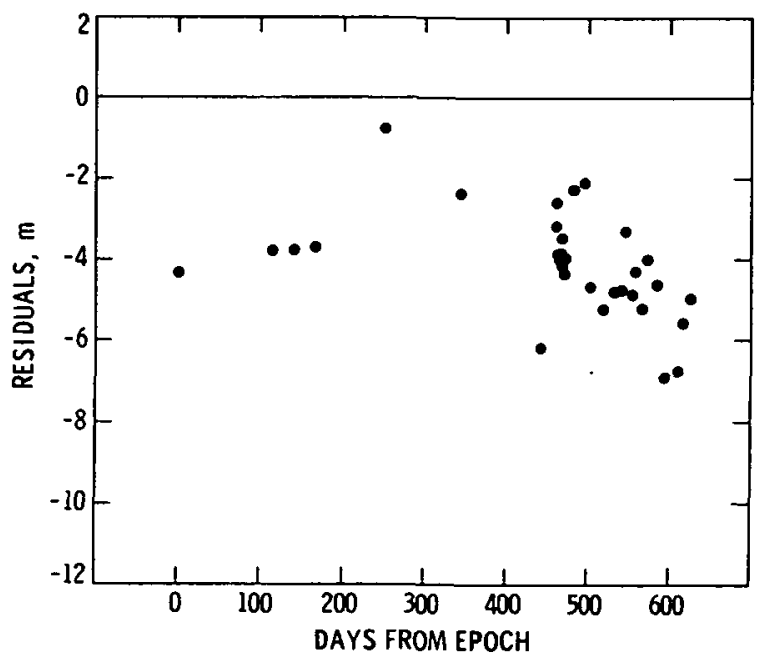

Fig. 13. DDOR residuals passed through, S-G baseline

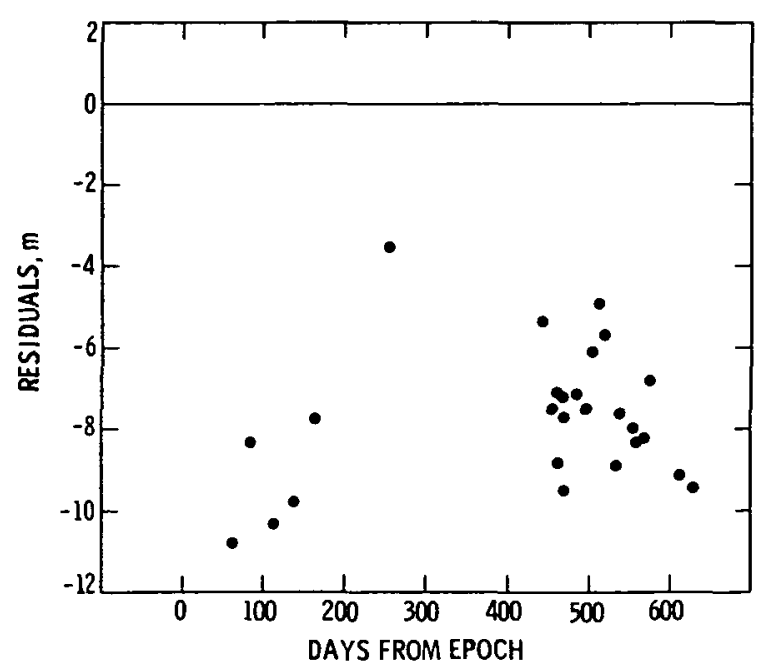

Fig. 14. DDOR residuals passed through, G-A baseline

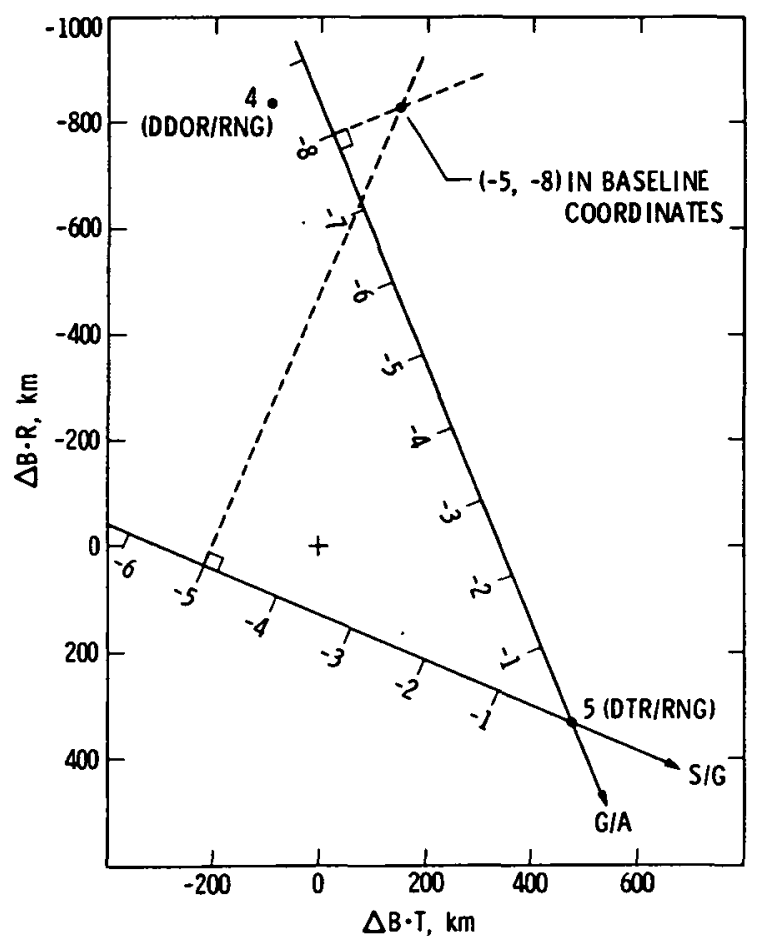

Fig. 15. Baseline residuals resolved into B-plane coordinates 


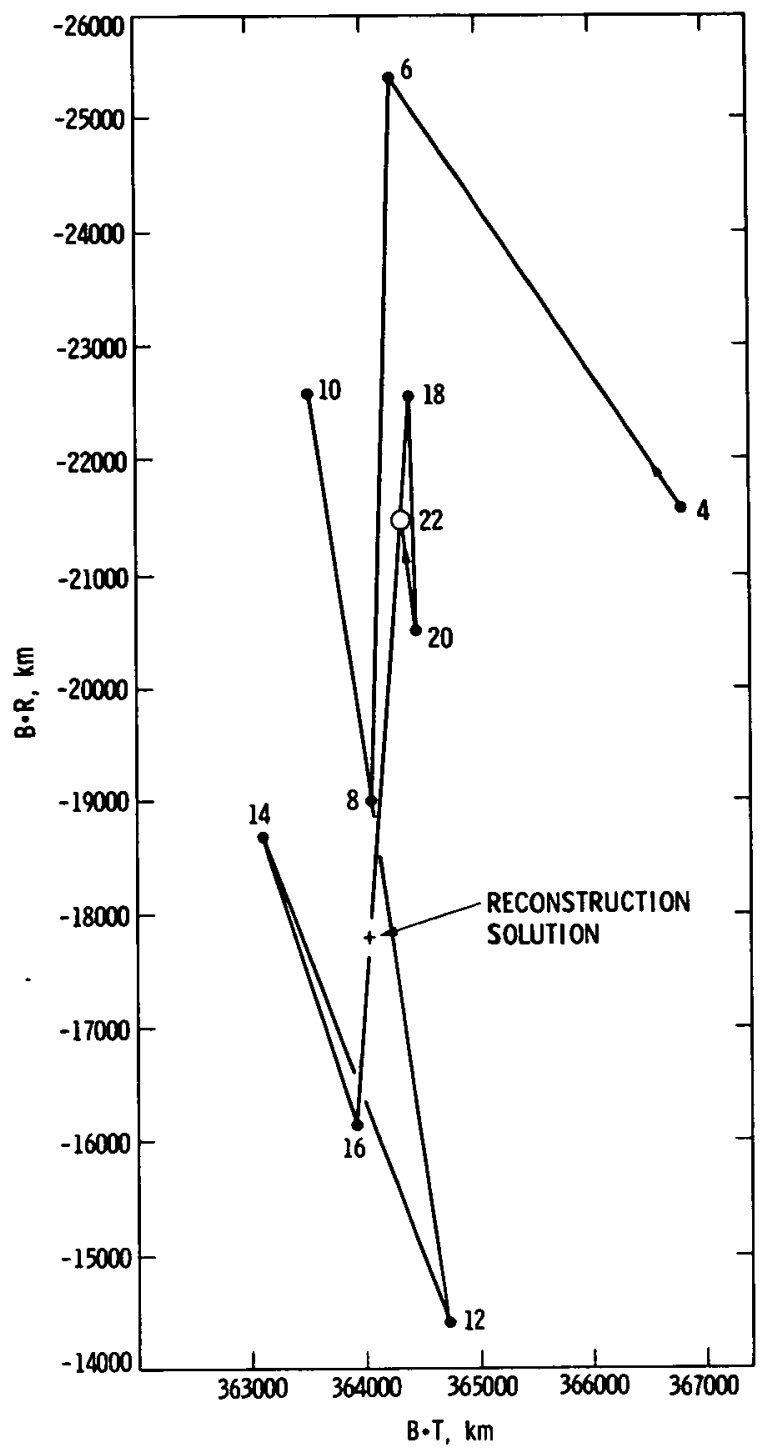

Fig. 16. B-plane history, doppler-range

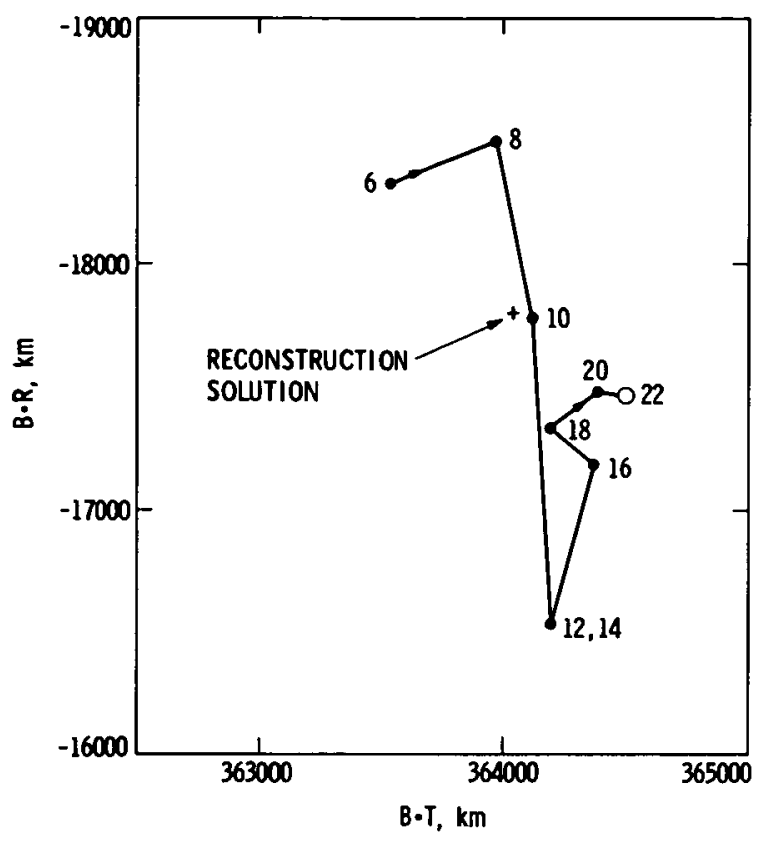

Fig. 17. B-plane history, DTR-range

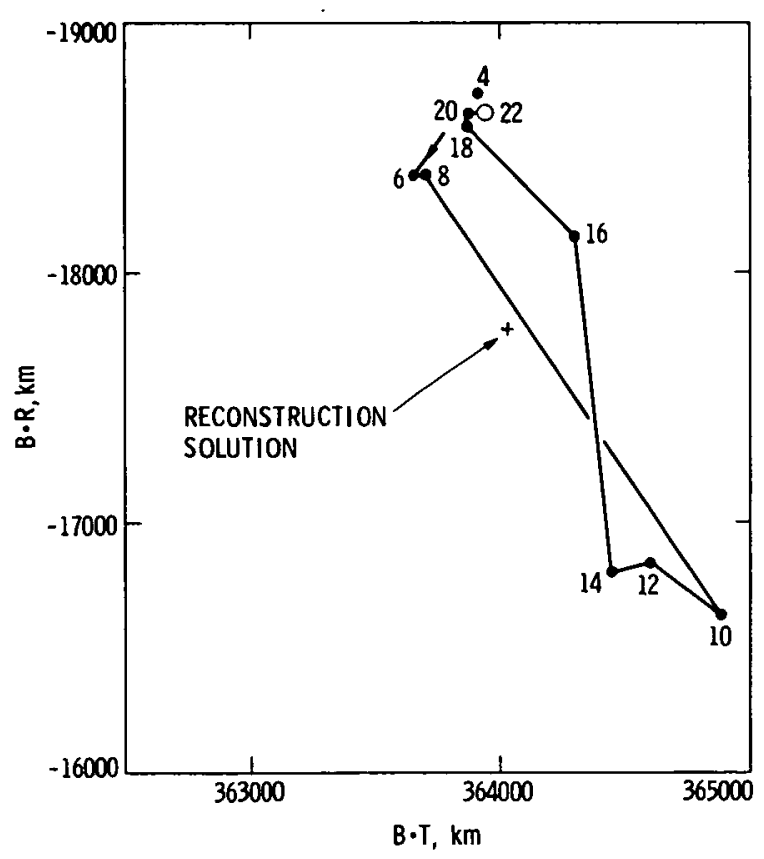

Fig. 18. B-plane history, DDOR-range 
ORIGINAL PAGE

OF POOR QUALITY

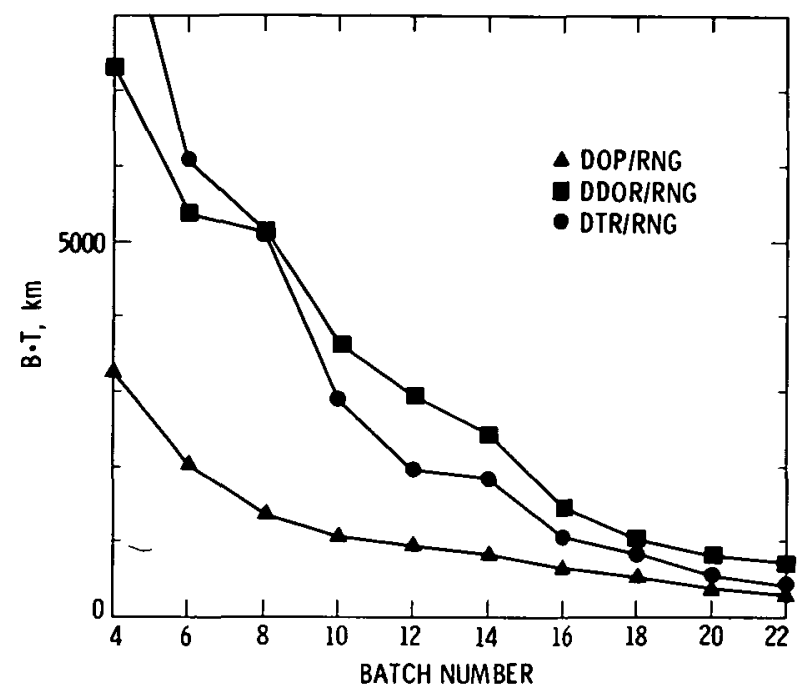

Fig. 19. Formal consider statistics in B.T.

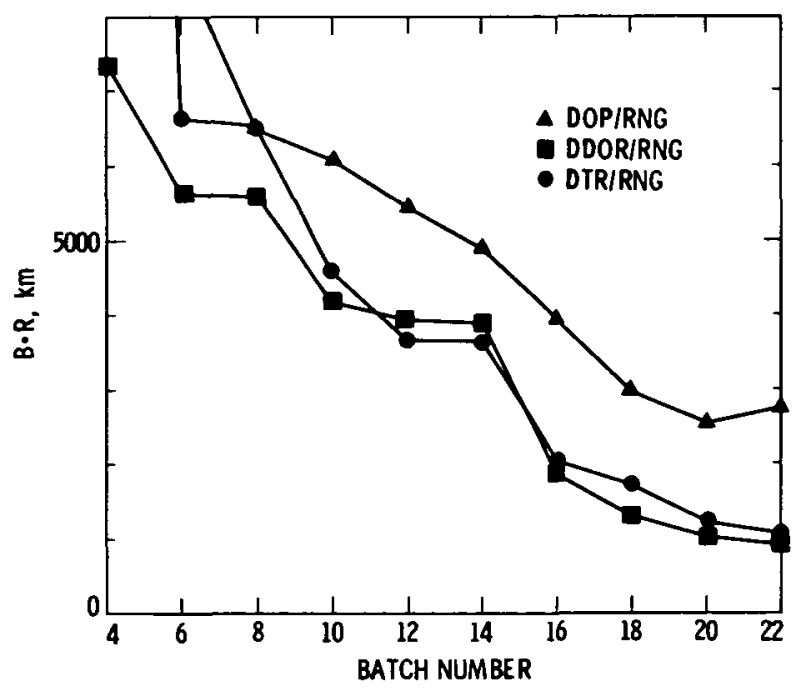

Fig. 20. Formal consider statistics in B.R. 